\title{
Philosophiques
}

\section{La question nationale}

\section{Michel Seymour}

Volume 19, numéro 2, automne 1992

Une nation peut-elle se donner la constitution de son choix?

URI : https://id.erudit.org/iderudit/027188ar

DOI : https://doi.org/10.7202/027188ar

Aller au sommaire du numéro

Éditeur(s)

Société de philosophie du Québec

ISSN

0316-2923 (imprimé)

1492-1391 (numérique)

Découvrir la revue

Citer ce document

Seymour, M. (1992). La question nationale. Philosophiques, 19(2), 5-26.

https://doi.org/10.7202/027188ar d'utilisation que vous pouvez consulter en ligne.

https://apropos.erudit.org/fr/usagers/politique-dutilisation/ 


\title{
LA QUESTION NATIONALE
}

\author{
par \\ Michel Seymour
}

Le présent numéro est entièrement consacré à la question nationale et il rassemble les Actes du Colloque intitulé « Une nation peut-elle se donner la Constitution de son choix? » qui a eu lieu les II et 12 mai 1992 à l'Université de Montréal dans le cadre du soixantième Congrès de l'Acfas. Il n'est sans doute pas nécessaire d'expliquer les raisons qui justifient une telle initiative ou de situer la problématique d'ensemble. Le contexte politique actuel foumit amplement de réponses à ces questions. Cette introduction pourrait alors n'être que l'occasion de présenter les différents auteurs et ne servir qu'à résumer brièvement leurs contributions. Mais on ne peut pas traiter la question nationale comme s'il s'agissait d'une question philosophique parmi tant d'autres. Elle nous « interpelle » aussi en tant que citoyen à part entière et on ne peut faire semblant d'intervenir sur un tel sujet de façon purement désintéressée. Il convient en ce sens de ne pas faire une Introduction au sens ordinaire et de proposer un préambule qui, malgré sa forme quasi-pamphlétaire, mettra en évidence les raisons profondes ayant motivé la mise sur pied de ce colloque et la publication de ses Actes.

\section{I}

Nous avons attendu plusieurs années avant de connaître la position constitutionnelle que le Gouvermement du Québec allait adopter et avant que l'actuel premier ministre du Québec clarifie enfin ses idées. Mais l'attentisme n'est pas toujours de bon aloi. Le vague, l'ambiguitté, l'indécision, bref les marques de commerce de celui qui nous dirige, ne l'ont d'ailleurs pas placé là 
où il se trouve par le plus pur des hasards. Ce sont les citoyens du Québec et, parmi ceux-ci, les intellectuels qui, par leur individualisme, leur indifférence, leur apolitisme désabusé ou leur corporatisme intéressé, ont contribué à ce qu'un tel politicien prenne en main les destinées du Québec pendant une période critique de son histoire.

Un système démocratique digne de ce nom ne peut se satisfaire d'un régime parlementaire dans lequel, lors de la période de questions à l'assemblée nationale, un premier ministre s'amuse avec les questions de l'opposition. Il doit donner lieu à des débats d'idées, permettre d'aller au fond des choses. Ceux qui ont la responsabilité de diriger les destinées d'un peuple doivent manifester un maximum de transparence, rendre des comptes à la population, expliquer leurs politiques et ne pas être complices des cyniques qui ne prennent pas au sérieux les affaires de la Cité. Or c'est exactement l'inverse qu'a fait le premier ministre jusqu'à présent. Au fil des ans, il a pu naviguer ainsi entre la mer et l'eau douce, gouvernant le plus souvent à la petite semaine, mais adoptant aussi parfois le ton solennel des grandes occasions comme pour masquer son manque flagrant d'orientation politique. Avec le recul, on peut facilement déceler un pattern. Élu par une forte majorité en I970, le chef du gouvernement libéral adopta alors un comportement qui ne s'est jamais démenti depuis.

Cela s'est produit une première fois à la Conférence de Victoria en r97I. Comme en fait foi un vieil éditorial de Claude Ryan, à l'époque directeur du Devoir, que ce journal a de nouveau fait paraître récemment (incité en cela par Guy Laforest, qui collabore au présent numéro), monsieur Bourassa a longtemps hésité à l'égard de l'Accord de Victoria, repoussé les échéances et refusé jusqu'à la dernière minute d'adopter une position claire. Il rejeta finalement ces offres en cédant à la pression de son entourage pour ne pas se mettre à dos l'opinion publique.

Il adopta dans les années qui suivirent une attitude semblable concernant la question linguistique. Il se réfugia derrière la caution de l'attente du rapport de la Commission Gendron pour ne pas avoir à intervenir. Lorsqu'il le fit, ce fut encore une fois parce qu'il n'avait plus d'autres choix et ce fut pour accoucher d'une position qui, lorsqu'elle prit la forme d'une loi (la loi 22), ne fit qu'accentuer le mécontentement de part et d'autre, trahissant encore une fois un manque d'orientation politique. 
On retrouve le même genre d'absence de leadership et de conviction profonde à son retour au pouvoir en Ig85. Il renonça tout d'abord à sa promesse adressée à l'intention de la minorité anglophone de modifier les aspects de la loi concernant la langue d'affichage. Il refusa ensuite de prendre explicitement position sur la question linguistique en prétextant que celle-ci faisait l'objet d'une requête devant les tribunaux. Lorsque la Cour Supérieure du Québec invalida certains aspects de la loi roI, il trouva refuge dans un autre attentisme et s'en remit à la Cour Suprême. Cest seulement après qu'un jugement analogue de la Cour de dernière instance l'eut contraint que notre premier ministre, emporté par une vague qu'il ne contrôlait pas, ne pu faire autrement qu'adopter une nouvelle loi, la loi 178 qui, à force de vouloir comme toujours ménager la chèvre et le chou, finit par laisser tout le monde sur sa faim.

Les choses n'ont pas changé sous sa gouverne après l'échec de l'Accord du Lac Meech. Il se réfugia derrière un soi-disant respect du processus démocratique et nous invita instamment à attendre le dépôt du rapport de la Commission Bélanger-Campeau ainsi que le rapport que devait produire pour lui le comité du parti libéral présidé par Maître Jean Allaire. La vaste majorité des intervenants au sein de la commission Bélanger-Campeau qui se prononcèrent en faveur d'une position constitutionnelle précise adoptèrent clairement une position souverainiste. Les autres rèclamèrent le rapatriement de l'un ou l'autre des pouvoirs historiquement réclamés par le Québec. Le rapport Allaire, pour sa part, réclama le rapatriement d'une compétence exclusive dans plus d'une vingtaine de secteurs de l'activité gouvernementale. Monsieur Bourassa prit ses distances à l'égard de ces deux documents, soit par l'ajout d'addenda à la fin du rapport de la Commission Bélanger-Campeau, soit par une profession de foi fédéraliste au Congrès du Parti libéral suite à l'adoption du rapport Allaire. Ces gestes contribuèrent tellement à contrebalancer les positions précises qu'il faisait semblant d'appuyer, qu'elles ne firent qu'accentuer le climat général d'ambiguïté et à susciter davantage de perplexité.

Nous retrouvons une fois de plus les mêmes ingrédients dans la stratégie actuelle de notre Premier ministre. Tous les éléments du scénario de la première heure sont encore au rendez-vous. Ce n'est qu'une fois acculé au pied du mur qu'il accouche d'une position mi-figue mi-raisin. Il nous annonce tantôt de Bruxelles une question référendaire portant sur la souveraineté, assortie d'une union économique et d'un gouvernement 
supranational élu au suffrage universel. Il revient ensuite sur sa décision et prétend qu'il n'y aura pas de référendum sur la souveraineté. Il argue vainement que la loi I5o est compatible avec la tenue d'un référendum sur les offres fédérales, mais reconnait que, pour ce faire, la loi devra être amendée. Il laisse planer l'impression auprès des membres de son parti et de la population que le rapport Allaire constitue toujours une base de négociation mais, du même souffle, il laisse passer sans broncher (ou en laissant seulement à ses ministres le soin de le faire, ce qui, du point de vue de l'impact auprès de la population, revient à la même chose) l'adoption au fédéral des projets de loi C-13, C-62 et C-8I qui consacrent l'interventionnisme d'Ottawa. Il annonce qu'il n'a nullement l'intention de se présenter à la table de négociation constitutionnelle sans garantie de succès, mais tous les rapports confirment des échanges téléphoniques répétés avec le fédéral et avec les autres provinces. Il donne l'impression à l'un (Don Getty) d'être favorable au Sénat triple-E alors que l'autre (Bob Rae) croit qu'il s'y oppose. Il prétend en 1990 qu'il ne sera plus jamais question dorénavant de négocier à onze, mais il reconnaît ensuite être prêt à le faire pourvu que la « substance de Meech » soit assurée et se rend finalement au Lac Harrington sans l'avoir obtenue. Quand on lui demande de prendre position, il se réfugie derrière le paravent de la commission parlementaire chargée d'étudier les offres. Il prétend qu'il devra s'en remettre au Cabinet, à l' Assemblée nationale et aux diverses instances de son Parti.

Mais le premier ministre n'a-t-il pas en tête une orientation politique précise? Ne souscrit-il pas au modèle européen? Le pattern dans lequel il s'est retrouvé régulièrement depuis plus de vingt ans suggère au contraire que ses " opinions » ne sont en fait que des stratagèmes pour se maintenir en place, des idées trouvées à la sauvette, des trucs improvisés, des positions confectionnées de toute pièce, empruntées « à gauche et à droite », qui favorisent le statu quo par opportunisme politique. Quand ce n'est pas Léon Dion qui lui dicte sa conduite, C'est Jacques Delors. Le pattern décrit plus haut révèle que la stratégie du premier ministre masque une absence de conviction et cela permet de mettre en doute la crédibilité de son adhésion au modèle européen.

L'analogie que d'aucuns voudraient établir entre le Canada et l'Europe ne tient pas pour des raisons qui ont maintes et maintes fois été ressasseees, mais aussi pour bien d'autres. Tout d'abord les États membres de la CEE sont déjà des pays souverains et ils 
contrôlent à tour de rôle sa direction sur une base semestrielle. Mais il y a aussi le fait que le futur État supranational envisagé par les européens n'aura de contrôle qu'au niveau de la monnaie, de la politique financière et, si tout va pour le mieux dans le meilleur des mondes, de la politique étrangère, encore que de ce côté, il y ait loin de la coupe aux lèvres, si le passé est garant de l'avenir et qu'on se donne pour modèle l'attitude adoptée par les différents membres de la CEE lors de la crise du Golfe et l'éclatement de la Yougoslavie. Il faut aussi s'empresser d'ajouter que dans le meilleur des cas, l'union monétaire sera atteinte par une poignée de quatre ou cinq pays vers rggg et que l'Angleterre et l'Allemagne sont de plus en plus réticentes à ce qu'il y ait plus que des objectifs économiques dans le projet européen.

Monsieur Bourassa invoque constamment le fait qu'il ne peut $y$ avoir d'union économique sans union politique, mais il ne fournit jamais d'arguments pour étayer cette affirmation. Comme le faisait remarquer Jacques Parizeau, l'accord de Maastricht limite le rôle de la future Banque Centrale à un pouvoir d'intervention qui ne doit pas être affecté par des considérations politiques. Il est vrai que certains des pays membres veulent qu'en plus de l'union économique, il y ait une forme d'union politique. Mais le fait qu'il $y$ ait une union économique ne prouve pas qu'il doive y avoir une union politique. En outre, l'union politique visée semble se rapprocher davantage d'une confédération d'États souverains que d'un fédéralisme proprement dit. Est-ce cela que le premier ministre veut pour le Québec? La plupart des souverainistes seraient alors d'accord avec lui. Enfin, même dans l'hypothèse où le fédéralisme serait bon pour l'Europe, il ne s'ensuivrait pas que le modèle européen soit celui qui convienne au Canada. Le fait que le premier ministre se réclame du modèle européen révèle qu'il ne pense pas la spécificité de la situation canadienne. Il invoque seulement à sa rescousse une idée pour camoufler son manque de leadership politique.

De toute façon, les pays de l'est veulent se joindre à la communauté européenne et cela pourrait retarder le processus d'unification. Ensuite, le traité de Maastricht, qui doit toujours être ratifié par les assemblées législatives des douze pays membres de la CEE, a déjà été rejeté par les Danois et cela crée de nouvelles complications. L'Angleterre a de plus fait enlever la référence au fédéralisme pour caractériser le projet et ne se voit pas contrainte de la même manière que les autres pays à l'intérieur de ce nouveau régime. Au surplus, les défenseurs de 
Maastricht s'empressent régulièrement de faire valoir aux opposants que la ratification du Traité n'empiètera pas sur la souveraineté des pays membres. Tout cela fait qu'on ne sait pas de quoi l'Europe aura l'air dans les prochaines années. En se la donnant comme modèle, monsieur Bourassa montre qu'il n'a pas d'idées précises.

La seule analogie possible avec le modèle européen serait un éventuel parlement supranational nord-américain et il est difficile de voir comment cela pourrait aboutir à autre chose qu'à une hégémonie des Etats-Unis. Monsieur Bourassa veut-il d'une union monétaire avec les Etats-Unis et souhaite-t-il un tel gouvernement supranational? Ensuite, il se compare aux Europeens, mais il n'est nullement question en Europe, que je sache, d'autoriser une intervention de la CEE sur les affaires culturelles des pays membres, alors que lui défend le droit d'intervention du gouvernement fédéral en matière de culture et ce, bien qu'il ait revendiqué un jour la souveraineté culturelle. Ça n'était sans doute qu'un mot creux. Quand on pense la spécificité nord-américaine, on se rend vite compte que le danger qui guette cette démocratie est une uniformisation de la culture et son nivellement par le bas. Le danger est que l'on en vienne à ne pas accepter les différences. L'Amérique du Nord est composée de grandes entités politiques qui ont tendance à centraliser les pouvoirs décisionnels, à négliger les régions et à concentrer le développement économique dans certains grands centres au dépens des régions périphériques. Elle ne peut être comparée à l'Europe qui est caractérisée par une formidable diversité de langues et de cultures. Si pour cette dernière, la voie à suivre est une certaine forme d'union politique qui permettrait de contenir les excès nationalistes l'ayant conduit si souvent à des guerres fratricides, le danger qui guette l'Amerique du Nord est l'excès contraire, c'est-à-dire l'intolérance à l'égard des différences, des spécificités régionales.

Les Europeens ont beau être prêts à adhérer à Maastricht, ils ne sont tout de même pas dénués d'intérêts nationalistes, à commencer par les Allemands. Ces derniers endossent peut-être le modèle européen, mais cela ne les a pas empêchès de poser un geste nationaliste specticulaire en procédant à la réunification. Et pour être en mesure de la financer, ils maintiennent des taux d'intérêt élevés à l'encontre de la volonté des autres peuples européens. Les Anglais, pour leur part, n'ont cessé de manifester leur hostilité a l'égard de toute tentative de brimer leur souveraineté nationale. Les Français, jusqu'à un certain point, ne font 
que prolonger le rêve gaulliste d'exercer un leadership politique sur l'Europe entière. En outre, le projet de Maastricht lui-même s'explique en partie par la convergence d'intérêts nationalistes, dans la mesure où il s'agit pour les pays membres de contenir autant que possible l'hégémonie allemande et de préserver les èconomies nationales européennes contre les visées envahissantes des superpuissances japonaise et américaine. Mais quelles que soient nos convictions politiques en ces matières, il y a fort à parier que la vision bigarrée du premier ministre du Québec, prenant tour à tour la forme d'un fédéralisme rentable, de la souveraineté culturelle, d'une superstructure ou d'une souveraineté partagée, est moins le reflet d'un hèritage européen qu'un ensemble de slogans improvisés, alimentés de points de vue qui, lorsqu'ils ne sont pas carrément incompatibles entre eux, ne servent qu'à masquer un manque de conviction politique.

En comparant sans nuances le Canada à l'Europe comme le fait le premier ministre, il prouve qu'il est à court d'idées, qu'il est dans l'incapacité de penser sa propre situation, et qu'il favorise au fond le maintien du statu quo. Mais n'est-il pas au moins un fédéraliste sincère? Les remarques qui précèdent permettent d'envisager une autre explication de la raison pour laquelle il endosse le fédéralisme à tout prix. Il vante les mérites de ce régime, mais cest qu'il préfèrerait au fond maintenir le statu quo. S'il a proposé les « changements » de l'Accord du Lac Meech, c'était pour en finir avec les velléités autonomistes du Québec. Le statu quo est pour lui l'idéal parce que c'est la seule façon de se maintenir au pouvoir sans avoir à défendre des idées politiques. Plusieurs politiciens opportunistes recherchent le pouvoir pour le pouvoir et n'ont en tête que leurs ambitions personnelles. Ce n'est pas le cas de Robert Bourassa. Mais n'ayant pas de conviction politique profonde, il ne comprend pas ceux qui s'engagent en politique pour réaliser un idéal et peut difficilement les représenter d'une quelconque façon. Pour se maintenir au pouvoir, il doit alors chercher à neutraliser ceux qui sont habités par un idéal quelqu'il soit. Pour gouverner, il compte sur le fait que les gens ne vont pas agir par conviction politique.

Cela a èté son erreur en 1987. En croyant en finir une fois pour toutes avec les ambitions autonomistes des Québécois, il s'est heurté à un nationalisme canadien grandissant, depuis le règne de Trudeau et l'adoption de la Constitution de ig82. Il a ouvert contre son gré une boite de Pandore. Son absence de conviction profonde explique aussi la raison pour laquelle il a cherché à tout prix d'essouffler, tout en feignant l'endosser, le 
nationalisme des Québécois suite à l'échec de l'accord du lac Meech. Il ne faut pas lui prêter d'intention machiavélique. C'est son incapacité viscérale à comprendre ceux qui sont animés d'une conviction profonde qui le pousse à agir ainsi. C'est aussi pour la même raison qu'il a accueilli les propositions du 7 juillet en disant qu'il 's'agissait d'un progrès considérable alors que toute la classe politique québécoise l'a rejeté en bloc. C'est enfin ce qui explique pourquoi il n'a cessé de rabaisser ses exigences. Après l'échec de Meech, il annonça qu'il n'y aurait plus de négociations à onze. Plus tard, il prétendit qu'un retour à la table de négociation ne surviendrait que s'il y avait «chance de succès ». Il finit par demander Meech, puis la « substance » de Meech, puis trois conditions, pour ne se contenter finalement que d'une et demie. Il affirma son opposition à un sénat égal pour se raviser par la suite. Qu'il s'agisse de la société distincte, de la justiciabilité du principe de l'autonomie gouvernementale autochtone ou de la limitation du pouvoir fédéral de dépenser, il n'y a décidément pas un seul domaine dans lequel il n'est pas disposé à faire des compromis.

Monsieur Bourassa ne demande pas mieux que d'ignorer la différence québécoise. Si jamais il soutient une position contraire au statu quo, c'est qu'il sera porté par un courant politique qu'il ne peut contrôler. Les nouveaux défenseurs du statu quo comme le professeur Stéphane Dion sont au fond ses alliés les plus proches. Leur argument en faveur du statu quo est nouveau. Face à l'impasse, au cul de sac constitutionnel, la seule solution qui reste est de prétendre qu'il s'agit d'un faux problème. Impossible d'accomoder la différence québécoise au sein de la fédération? Qu'à cela ne tienne, il nous reste encore une porte de sortie. Cette porte de sortie la voici: le Québec n'est de toute façon pas réellement différent $\mathrm{Car}$, en effet, quelle est la différence québécoise? Une différence de langue? Une différence de culture, ne serait-ce qu'au sens où les Québécois subissent autant l'influence de la France que celle des Etats-Unis? Un code civil? Une différence au niveau de la reconnaissance québécoise des droits collectifs alors qu'au Canada anglais, on critique les lois linguistiques, on résiste à une reconnaissance substantielle de la société distincte et on adopte une loi référendaire individualiste qui contraste singulièrement avec celle du Québec? Une différence dans la politique économique révélée entre autres à l'occasion du débat autour du libre-échange? Une différence d'intérêts économiques fondée sur la volonté de développer dans la région de Montréal un centre économique un tant soit 
peu comparable à celui de Toronto? Une diffèrence de visions de ce que doit être le Canada, perpétuée depuis plus de trente ans par les «centralisateurs » canadiens et les « décentralisateurs » québécois, les défenseurs trudeauistes de l'État-nation canadien et les défenseurs du Canada multinational? L'existence de deux solitudes? De l'avis de nos nouveaux admirateurs du statu quo, tout cela n'aura été qu'une illusion!

On ne saurait sous-estimer l'importance de ce qui s'est produit en 1982. On mentionne habituellement le fait que le Canada anglais a rapatrié unilatéralement la Constitution dans le dos du Québec et que ce dernier a alors perdu son droit de veto. Mais le plus grave est la mise sur pied de l'État-nation canadien et le déclin de l'idée des deux peuples fondateurs. L'adoption d'une Charte des droits individualiste enchâssée dans la Constitution a en effet eu non seulement pour conséquence d'entraîner une judiciarisation du politique, mais aussi de consacrer l'uniformisation de la " nation canadienne » et de rendre la loi ror anticonstitutionnelle. L'accord du Lac Meech représentait une faible tentative de renverser la vapeur, mais il a été rejeté. Le Canada anglais cherche maintenant à consolider son État-nation en constitutionnalisant, par la réforme du sénat, un rẻgime politique dans lequel toutes les provinces sont égales entre elles et où aucune place n'est laissèe à l'idée des peuples fondateurs.

La seule diffërence est que les canadiens anglais se sont rendu compte qu'ils avaient intérêt à reconnaître symboliquement les droits collectifs dans la Constitution. Il se sont en ce sens vite emparé de la question autochtone parce qu'elle comportait entre autres choses plusieurs avantages politiques. Elle permettait de se donner bonne conscience à l'égard des revendications nationales des communautés vivant sur son territoire, de mettre dos à dos les nationalismes québécois et autochtones, de les considérer sur un pied d'égalité et de les jouer l'un contre l'autre. Autrement dit, on a cherché à faire une démonstration par l'absurde de l'idée d'une souveraineté politique québécoise en suggérant que cela devrait aller de pair avec un même genre de statut pour les autochtones. Les autochtones ont alors euxmêmes emboîté le pas dans la dénonciation des désirs autonomistes du Québec pour se faire du capital politique auprès du Canada anglais.

Le Québec a aussi bénéficié des faveurs d'une reconnaissance toute symbolique par l'admission d'une clause de société distincte. Mais celle-ci s'est vue restreinte dans sa signification et 
contrainte dans son application. Elle s'est retrouvée noyée dans une clause Canada et nier par la réforme du Sénat. Elle ne peut plus désormais être interpréte comme pouvant autoriser un nouveau partage des pouvoirs et, par-dessus le marché, elle peut désormais être abrogée par sept provinces représentant plus de la moitié de la population. Le fait essentiel demeure donc la consolidation de l'État-nation canadien. Que le sénat envisagé soit equitable ou egal, il ne fait que renforcer le pouvoir central au dépens du pouvoir provincial. Or en manifestant son accord de principe avec un sénat égal, en se contentant d'une clause symbolique de société distincte et en reléguant la question du partage de pouvoirs à des ententes bilatérales, monsieur Bourassa souscrit implicitement au renforcement de l'État-nation canadien. Il montre encore une fois qu'il veut à tout prix en finir avec la différence québécoise.

La « manière Bourassa » ainsi systématiquement pratiquée sur une grande échelle et sur des sujets d'une gravité exceptionnelle ne rend pas service au Québec. Elle finit par s'avérer antidémocratique puisqu'elle table sur la lassitude et le cynisme politique. Il est scandaleux de voir l'actuel premier ministre s'affranchir de ses responsabilités démocratiques et voguer comme bon lui semble sur la haute mer constitutionnelle en faisant fi de la commission Bélanger-Campeau, du rapport Allaire, de son Parti, de la loi 150, de plus de 700,000 signatures réclamant un référendum sur la souveraineté avant le 26 octobre 1992 et des sondages qui révèlent depuis plus de deux ans l'existence d'une majorité souverainiste. Quelle que soit l'issue de l'aventure constitutionnelle actuelle, il reste que le premier ministre cherche constamment à éviter les prises de position et à ne prendre de décision que lorsqu'il se voit absolument contraint de le faire. La position qu'il prend en définitive est toujours une vision hybride qui, à force de vouloir réconcilier les contraires, finit par rendre tout le monde insatisfait

Nous avons tous à assumer la responsabilité d'un tel état de choses. La plus grande responsabilité est peut-être celle des intellectuels qui n'ont pas suffisamment su imposer un régime de discussion des idées, créer des conditions favorables à une remise en question perpétuelle du gouvernement en place, soit pour endosser ou rejeter ses politiques. Exception faite du Devoir auquel il nous faut rendre hommage et de quelques interventions isolées, l'absence des intellectuels sur la scène politique est en partie la cause du vacuum dont profite l'actuel premier ministre. 


\section{II}

Cest pour contribuer modestement à combler en partie ce vide que le présent numéro spécial de Philosophiques a été conçu. Il s'agit donc d'une intervention qui, par son existence même et par le moment choisi pour se manifester, a un caractère éminemment politique. Cela ne l'empêche pas de se situer bien au-delà d'une simple prise de position partisane et de réunir des textes dont les visées sont, pour la plupart, d'abord et avant tout "scientifiques ». Cependant, certains orateurs ont été explicitement invités à se prononcer sur les enjeux constitutionnels actuels et il ne faut pas se surprendre de trouver, çà et là, des interventions qui prennent davantage la forme de prises de position. Il m'a en effet semblé qu'il ne fallait pas, à l'occasion de ce numéro spécial, s'en tenir à un format parfaitement «académique ».

L'idée d'organiser un Colloque sur la question nationale est issue à l'origine de discussions virulentes avec mon collègue Lukas Sosoe. Il nous a alors semblé opportun d'inviter les philosophes et politologues d'ici et de l'extérieur du Québec à se prononcer sur la question nationale. On trouvera dans les pages qui suivent les textes des communications qui furent présentées lors du Colloque. Le lecteur pourra avoir accès à des textes qui exploitent à peu près tout l'éventail des positions philosophicopolitiques possibles.

Les diverses interventions ont été rassemblées en trois groupes distincts. Dans une première partie, il est principalement question de la définition du concept de nation et du nationalisme proprement dit et on y retrouve les textes de Sosoe, Couture, Dufour et Seymour. Dans la deuxième partie, il est surtout question de la Constitution et du problème posé par le pluralisme culturel. Les textes de Leydet, Kymlicka, Weinstock et Legault gravitent tous autour de cette problematique. La troisième et dernière partie rassemble des réflexions ayant trait aux conditions d'accession à la souveraineté dans le cadre d'une philosophie d'inspiration libérale. Les contributions de Buchanan, Nielsen, Caldwell et Laforest se concentrent sur cette thématique.

Lukas Sosoe ouvre le bal avec un texte provocateur qui cherche à accentuer l'opposition entre, d'une part, un nationalisme fondé sur l'identité linguistique, l'histoire et la tradition et, d'autre part, une approche contractualiste qui est au fondement de la modernité. Sosoe est d'avis que le Québec a depuis 
toujours appuyé sa démarche exclusivement sur la promotion de la nation définie à partir de la culture, la tradition et l'histoire. Il lui parait en ce sens problématique, voire même incohérent, de se fonder sur un tel nationalisme pour négocier un nouveau contrat avec l'État fédéral. En particulier, la revendication d'une socièté distincte dans le cadre d'une entité républicaine comme le Canada «n'est pas compatible avec la nature romantique de l'identité québécoise $»$. Pour Sosoe, l'ordre démocratique moderne est par essence individualiste et ne peut admettre des facteurs identitaires reposant sur des visions romantiques, historicistes et donc pré-modernes. Il s'oppose aux théories communautariennes de M. Sandel, C. Taylor et A. McIntyre et critique les tentatives vaines de Buchanan et Kymlicka d'accorder une place aux droits collectifs. De telles approches finissent toujours par admettre sans le démontrer l'existence de tels droits ou à reconnaître leur réductibilité à des droits individuels. L'auteur remet en question les approches qui visent à rendre compatibles l'idée d'un Etat libéral démocratique avec l'admission de droits collectifs et soutient que la souveraineté est non-négociable en ce sens qu'elle ne peut faire l'objet d'une justification rationnelle.

Jocelyne Couture s'interroge ouvertement sur la question servant de thème au Colloque. Elle fait valoir qu'en un sens, il est analytique de prétendre qu'une nation puisse se donner la Constitution de son choix. Par définition, une nation est une entité ayant une expression politique et elle n'existe que dans la mesure où elle s'affirme politiquement en se donnant, entre autres choses, une constitution. Mais du même coup, une nation n'a pas le choix en cette matière et voilà pourquoi il faut répondre par la négative à la question de savoir si une nation peut se donner la Constitution de son choix. Elle s'en prend aux «penseurs de la vérité » qui, comme Rousseau, cherchent à définir la nation par des traits empiriques et la conçoivent indépendamment de la capacité de se donner une expression politique. Mais elle récuse tout autant les « penseurs de l'efficacité » qui, comme Hobbes, conçoivent les Etats indépendamment des populations gouvemées. Pour Couture, les nations doivent d'emblée être des États-nations. Or une nation sans Etat, lorsque conjointe à un Etat sans nation, ne fait pas un Etat-nation. Les penseurs de la vérité et ceux de l'efficacité, bien qu'opposés en apparence, pratiquent tous les deux « l'art de la séparation » des pouvoirs politiques et des communautés nationales. Ainsi la nation conçue à partir d'une définition purement culturelle et 
folklorique peut très bien cohabiter avec un pouvoir politique qui ne serait pas l'expression politique d'une communauté nationale. Couture dénonce cette collusion entre les penseurs de la vérité et ceux de l'efficacité. Il faut selon elle adopter plutôt le point de vue de Weber et fonder la légitimité des pouvoirs publics sur la croyance en cette légitimité. Il faut admettre que les nations se caractérisent à partir d'un ensemble de croyances partagées par les membres d'un groupe donné. La nation n'existe que si un ensemble d'individus y croient.

Christian Dufour s'emploie à vanter les mérites du concept de « société distincte » qui lui apparaît prometteur pour le Québec. Il s'agit tout d'abord d'une notion qui fait consensus dans toute la classe politique québécoise. Il faut en effet selon lui être de mauvaise foi pour nier le fait que le Québec constitue à tout le moins une société distincte. La notion peut en ce sens rallier libéraux et péquistes. En outre, il s'agit d'un concept moderne qui ne réduit pas le Québec à sa composante culturelle. Son contenu est minimal et c'est pourquoi il permet de rallier des positions sensiblement différentes. Malgré ce caractère minimal, la notion est intimement liée à une frontière et va de pair avec le respect de l'intégrité territoriale du Québec. Son application va ensuite à l'encontre de l'orthodoxie libérale qui ne reconnaît que l'existence des droits individuels. La notion est exportable et le Québec devrait se vanter d'être le premier à avoir cherché son implantation comme il le fait depuis cinq ans. Enfin la promotion de la société distincte s'avère un atout indispensable sur le plan stratégique. Pour exercer un véritable rapport de force politique, il convient de s'appuyer sur une notion qui permet de ratisser large. Elle permet aussi par son caractère modéré de nous attirer la sympathie des Américains, comme ce fut le cas suite à l'échec de l'Accord du Lac Meech. Mais surtout elle est compatible avec le fait que le Québec puisse accéder à la souveraineté politique.

Michel Seymour propose une justification du nationalisme qui s'appuie en partie sur des thèses issues de la philosophie de la psychologie. La doctrine anti-individualiste qu'il met de l'avant comportent trois volets essentiels: une thèse concernant la nature linguistique des contenus de pensée; une thèse fondée sur les expériences de pensée de Hilary Putnam et Tyler Burge, à l'effet de considérer que l'environnement socio-linguistique contribue à l'individuation des contenus de pensée; enfin, une thèse générale sur l'impossibilité des langages privés inspirée de Wittgenstein. Puisque la communauté socio-linguistique contribue 
à l'identité des personnes, la promotion et la protection des droits de la personne devraient, si l'anti-individualisme est admis, aller de pair avec la protection et la promotion des droits de la communauté linguistique à laquelle elle appartient. Lorsque la communauté linguistique est une nation, comme cela est indéniablement le cas pour le Québec, il est possible de montrer ensuite à quelles conditions l'anti-individualisme peut donner lieu à une définition minimale et positive du nationalisme et comment on peut s'en servir aussi, comme Charles Taylor l'admettait il y a plus de douze ans, pour justifier l'accession du Québec à la souveraineté politique. Après plus de trente années d'échecs constitutionnels, il devient rationnel de proposer la souveraineté comme moyen de promouvoir et protéger la nation québécoise.

Dominique Leydet examine les textes de Jürgen Habermas écrits à l'occasion du débat des historiens des annèes 80 et de la réunification de l'Allemagne. Elle analyse plus particulièrement sa notion de patriotisme constitutionnel. Elle montre comment la critique habermasienne de la restauration du préjugé par Gadamer, la nécessité de préserver le pluralisme, la revendication de l'héritage critique des Lumières et la nécessité kantienne de formuler une éthique universaliste, interviennent dans l'élaboration de son point de vue et contribuent à.faire basculer la question de l'identité nationale de la sphère de la culture à la sphère juridico-politique. Ainsi le patriotisme constitutionnel de Habermas est un nationalisme épuré de toute référence à l'histoire, à la tradition et à la culture et prend plutôt la forme d'une adhésion à un ensemble de principes universels spécifiquement appliqués à un groupe donné, ce qui rappelle la notion hégélienne de l'universel concret. Tout cela est possible pourvu que les communautés faisant partie intégrante d'un pays donné soient suffisamment culturellement homogènes et permettent l'adhésion à un même ensemble de principes universels. Le patriotisme constitutionnel n'interdit pas la reconnaissance des droits collectifs dans une constitution, mais il requiert, dans l'éventualité d'un Québec souverain, la préséance de la Charte québécoise des droits et libertés sur la charte de la langue française.

Will Kymlicka distingue les États multinationaux qui résultent de la cohabitation de différentes cultures au sein d'un même pays et les Etats polyethniques qui résultent de l'immigration. Il prétend que la plupart des états souverains sont multinationaux ou polyethniques. Il critique le point de vue de N. Glazer 
qui considère que l'Etat doit répondre avec une " neutralité bienveillante » aux aspirations culturelles et le point de vue de Knopff qui recommande aux gouvernements de légiférer en matière linguistique en mettant l'emphase sur le caractère utilitaire, culturellement neutre, du langage. Selon Kymlicka, la tradition libérale de L.T. Hobhouse, J.S. Mill et T.H. Green a fait valoir une variété de points de vue à l'égard des minorités que la présente orthodoxie libérale tend à obscurcir. Il s'interroge ensuite sur les raisons qui font que la pensée néo-libérale de l'après-guerre s'est montrée réticente à reconnaitre les droits des minorités et à admettre le pluralisme culturel. Contrairement à Sosoe, il ne croit pas que cela repose sur une conception individualiste qui serait un trait inhérent à toute pensée libérale et il admet la possibilité de reconnaitre l'existence des droits collectifs à l'intérieur d'un tel cadre. Il fait reposer plutôt son explication sur des facteurs historiques survenus principalement aux Etats-Unis, mais qui ont influencé tous les penseurs libéraux. La peur de voir dégénérer la paix internationale, l'engagement à l'égard du principe de l'égalité des races et les inquiétudes suscitées par les demandes toujours de plus en plus grandes des groupes d'immigrants, expliquent en partie le retard des penseurs néo-libéraux à reconnaître les phènomènes multinationaux ou polyethniques.

Daniel Weinstock questionne la possibilité que puissent cohabiter le nationalisme et le pluralisme culturel. Il fonde son approche sur une pensée résolument communautarienne. Même si la thèse politique communautarienne selon laquelle l'Etat a une responsabilité à l'égard des intérêts culturels des individus ne découle pas automatiquement de la thèse psychologique à l'effet que l'individu est en partie défini à partir de son attachement à une communauté culturelle, il se porte à la défense des deux et cherche à formuler les conditions qui feraient en sorte d'admettre la responsabilité pour l'Etat de prendre en charge les identités culturelles des communautés qui le constituent Il s'inscrit en faux contre la thèse défendue par Kymlicka selon laquelle rien dans la pensée néo-libèrale n'interdirait en principe la reconnaissance des postulats communautariens évoqués plus haut Il montre en particulier comment la théorie de Rawls est intimement liée à des postulats individualistes et comment, pour cette raison, elle est difficilement conciliable avec une telle reconnaissance. Mais cela n'implique pas que le liberralisme soit en soi incompatible avec le communautarisme. Il termine en ce sens son article en cherchant à distinguer une approche 
procédurale du libéralisme, fondée sur l'adoption de critères formels normatifs gouvernant l'interaction des groupes et dégagée de toute forme d'adhésion à une idéologie individualiste, d'une approche substantielle qui s'inspire d'une telle idéologie et qui se prononce malencontreusement sur la nature des individus, citoyens et sociétés.

Josée Legault prend le parti de s'attaquer à une question d'une gravité insoupçonnée, celle de l'enchâssement d'une Charte des droits et libertés dans un éventuel Québec souverain. Elle s'emploie à mettre en évidence les effets pervers encourus par le fait d'avoir voulu procéder de la sorte en ce qui a trait à l'insertion d'une Charte dans la Constitution canadienne de 1982 et prêtend que les mêmes problèmes apparaittront avec la Charte québécoise une fois que le Québec aura fait sécession. Elle montre comment un tel enchâssement a pour effet de déresponsabiliser les pouvoirs politiques et d'augmenter proportionnellement le pouvoir des juges non élus. Ensuite, étant donné le caractère individualiste de la Charte québécoise, cela pourrait conduire à une remise en question par les tribunaux des différents aspects de la Charte de la langue française. Elle montre aussi que la clause dérogatoire, loin d'instaurer un équilibre, introduit une contradiction au coeur même de la Constitution. On ne saurait davantage être rassuré par l'article i de la Constitution qui prévoit les exceptions à une application sans restriction des principes gouvemant les droits individuels fondamentaux, car cet article est assorti du fameux test Oakes qui est extrêmement complexe dans son application et incompréhensible dans son interprétation. Elle souligne aussi le fait que l'égalitarisme individuel géré par des tribunaux entrainerait une centralisation des pouvoirs qui ne respecterait plus la diversité sociologique. Ces conséquences sont d'autant plus graves que l'accès à la justice va s'avérer onéreux pour le simple citoyen. Elle s'inquiète en conclusion de la disparition de la culture politique qui risquerait de s'en suivre et de l'évacuation des luttes de pouvoirs politiques désormais médiatisées par la représentation des technocrates du droit Le citoyen ne serait plus au service de son pays mais de ses institutions juridiques.

Allen. Buchanan s'interroge sur l'existence d'un droit juridique (constitutionnel ou international) pour l'accession à la souveraineté politique et répond par la négative. Il croit cependant que les peuples peuvent se réclamer d’un droit moral pour procéder à la sécession, mais il estime qu'ils doivent alors s'appuyer sur des raisons solides, comme le fait d'empêcher la 
destruction de leurs cultures. A son avis les Québécois invoquent plutôt comme argument le désir de pouvoir orienter comme ils l'entendent le développement de leur propre culture et cela lui apparait insuffisant. Tout en reconnaissant que les nations n'ont de toute façon pas besoin d'invoquer un droit quelconque pour faire sécession, il termine son article en posant des questions relatives aux conditions dans lesquelles la sécession peut être réalisée. Il pose en particulier le problème de l'intégrité territoriale du Québec et celui de la reconnaissance des communautés autochtones. Il prétend que les deux questions sont inextricablement liées et que la résolution du premier problème requiert qu'une solution soit aussi apportée à l'autre. Même s'il n'y a aucun statut juridique justifiant l'intégrité du territoire québécois, car son territoire actuel est celui qu'il a en tant que province du Canada, il reconnait cependant que le Québec pourrait avec raison s'attendre à avoir droit au même territoire une fois que la sécession serait un fait accompli. Il faut toutefois qu'une renégociation de la situation des peuples autochtones fasse partie intégrante du processus d'accession à la souveraineté politique. Il croit en particulier qu'il serait fallacieux de prétendre que les seules options qui s'offrent aux communautés ethniques sont, soit la souveraineté pleine et entière, soit le rabaissement au statut de minorité culturelle.

Kai Nielsen répond par l'affirmative à la question de savoir si les peuples ont un droit inhérent à l'auto-détermination. Si un peuple se perçoit comme ayant une culture et une tradition distinctes et constitue un groupe majoritaire sur un territoire distinct, il peut faire sécession de l'Etat-nation dans lequel il se trouve pourvu qu'il ne viole pas les droits des individus vivant sur son propre territoire. Il critique ceux qui, comme Marx ou Comte, ont voulu faire fi de l'attachement des individus à leur propre culture et leur rétorque que cet attachement joue un rôle dans l'identité de ces mêmes individus. Il fait aussi écho aux critiques de Mandelbaum et Lukes à l'égard de l'individualisme méthodologique. Il critique ensuite ceux qui, tout en reconnaissant cette détermination socio-culturelle sur l'identité des personnes, prétendent que les facteurs identitaires ne sont pas intrinsèquement bons, contrairement à la liberté, au bonheur ou à la satisfaction des désirs. Il fait valoir que nous les considérons bons et qu'il n'y a de toute façon aucune autre justification possible. Enfin, en comparant la sécession au cas du divorce entre individus, il soutient qu'il n'est pas juste, comme Buchanan le prétend, de suggérer que le fardeau de la preuve repose sur les 
épaules des sécessionnistes. De la même manière qu'un divorce entre deux êtres peut survenir du fait que l'un ne veut tout simplement plus vive avec l'autre, un peuple peut choisir de faire sécession. Le fardeau de la preuve est entièrement du côté de ceux qui veulent $s$ y objecter.

Gary Caldwell part du constat que le Québec constitue une société libre et démocratique où prévaut une très grande qualité de vie et un haut degré de justice sociale. Il s'emploie ensuite à étayer ces différents traits caractéristiques fondamentaux de la socièté québécoise. Il s'insurge contre ceux qui, comme Bercusson et Cooper, insinuent que le Québec est une société intolérante dans laquelle règne un collectivisme brimant les droits individuels de ses minorités. Plusieurs indicateurs permettent d'établir le contraire. Qu'il s'agisse de la participation aux élections, de la loi électorale, de l'ouverture à l'égard de l'immigration, du taux de mortalité infantile très faible et de celui concernant l'espérance de vie, ou encore de l'absence de violence raciste, le Québec occupe une position très enviable à l'échelle mondiale. Le corpus de coutumes et de conventions de la société civile québécoise ainsi que sa culture politique contribuent à former la Constitution de la société, au sens oú E. Burke et A de Tocqueville entendent cette expression. Caldwell s'estime donc en droit de prétendre qu'en un certain sens, le Québec a déjà une Constitution. Il affirme en outre que le rapatriement de la Constitution de 1982 constitue un geste anti-constitutionnel du point de vue du Québec. Non seulement parce que cela s'est fait sans son consentement mais aussi parce qu'elle consacre l'abandon de la politique parlementaire et rend illégitime la loi Ior. En ces circonstances, le Québec doit mettre en demeure le reste du Canada de reconnaittre l'erreur de 1982 et de renoncer à la Constitution qu'il adopta alors. Sinon, qu'il se sépare du reste du Canada, c'est-à-dire du Québec.

Guy Laforest reprend en le transformant le mot de Mackenzie King: pas nécessairement la sécession, mais la sécession si nécessaire. Il pose tout d'abord un diagnostic de crise à l'égard du régime fédéral canadien en s'inspirant d'ètudes rassemblées dans des recueils rendant hommage à deux des piliers de la science politique canadienne et québécoise: Gérard Bergeron et Léon Dion. S'appuyant sur certaines des contributions de politologues anglo-canadiens comme J. Meisel, D. Smiley ou B. Neatby, il remarque que plusieurs indicateurs laissent entrevoir la fin du pays conçu sur le modèle de la dualité des peuples fondateurs. Puis il propose en terminant un argument en faveur de 
la sécession qui s'inscrit dans le cadre d'une èthique libérale. Trois facteurs sont invoqués à l'appui de cette option. Il y a d'abord et avant tout le fait que la révision de la constitution canadienne s'est faite en r 982 sans l'appui du gouvenement du Québec et sans que la population du Québec n'ait été consultée par la voie d'élections générales fédérales ou par référendum. Il y a en deuxième lieu le fait que le Québec doive promouvoir et protéger sa propre culture. Il prétend que les cinq conditions imposées par Buchanan pour justifier la sécession sur la base de la protection de la culture sont satisfaites. Le statut du territoire du Québec peut être clarifiè et la sécession peut ne pas donner lieu à un gouvernement qui bafoue les principes libéraux. Le Québec a aussi épuisé tous les recours à l'intérieur du régime fédéral et sa culture est en péril, comme le confirme une série de faits empiriques. En dernier lieu, le Québec sera, selon Laforest, bien placé pour faire la sécession s'il peut se doter d'une Constitution plus généreuse que celle de 1982 et reconnaître l'existence de plusieurs communautés nationales sur son propre territoire.

\section{III}

Je voudrais remercier la Faculté des arts et des sciences et le département de philosophie de l'Université de Montréal d'avoir appuyé financièrement l'organisation du Colloque, ainsi que l'Association canadienne-française pour l'avancement des sciences qui a permis de subventionner en partie le déplacement de nos conférenciers étrangers. Jai pour ma part bénéficié de l'aide financière du Fonds FCAR, dans le cadre de son programme de subventions aux nouveaux chercheurs, et du Conseil de recherches en sciences humaines du Canada, dans le cadre de son programme de Bourses du Canada. Je remercie Louise Marcil-Lacoste, Hélène Pelletier-Baillargeon et Jean Roy d'avoir accepté de présider les séances du Colloque. Je m'en voudrais de ne pas mentionner la contribution de Suzanne Poirier qui m'a assisté lors du colloque et celle de Jean Lachapelle qui m'a aidé à préparer la publication des Actes. Geneviève Sicotte a assuré de manière très professionnelle la traduction des textes de Buchanan, Kymlicka et Nielsen. C'est à Bernard Gadoua que nous devons l'excellent travail de composition du texte. J'ai pu en outre bénéficier à plusieurs reprises des avis fort judicieux de Daniel Weinstock ainsi que de son appui moral. Je désire exprimer ma gratitude à l'égard des évaluateurs anonymes qui ont par leurs commentaires, corrections et suggestions, grandement contribué à améliorer l'ensemble des textes, et ce, malgré le peu de 
temps mis à leur disposition. Enfin, je remercie la revue Philosophiques et sa directrice Josiane Ayoub d'avoir bien voulu permettre la publication des textes du Colloque. Je dédie ces Actes à la mémoire de René Iévesque.

Département de philosophie

Université de Montréal

Allen Buchanan est professeur au département de philosophie de l'Université d'Arizona à Tucson. Il est l'auteur de Secession. The Morality of Political Divorce. From Fort Sumter to Lithuania and Quebec, chez Westview Press en 1990.

Gary Caldwell a été jusqu'à récemment affilié à l'Institut québécois de recherches sur la culture et il enseigne au département de sociologie et d'anthropologie de l'Université de Concordia. Il a notamment publiè Les anglophones du Québec. De majoritaires à minoritaires à l'IQRC en 1982 et National Survival in Dependent Societies: Social Change in Canada and Poland aux Presses de l'Université de Carleton en 1990.

Jocelyne Couture est professeur au département de philosophie de l'Université du Québec à Montréal et a publié Ethique et Rationalité chez Mardaga en 1992.

Christian Dufour est affilié à l'Institut de recherches politiques du Canada. Il a fait paraittre Is défi québécois aux éditions de l'Hexagone en 1989 et un ouvrage intitulé Pour en finir avec la Conquête aux Éditions Boréal en 1992.

Will Kymlicka est professeur au département de philosophie de l'Université d'Ottawa et a publié Liberalism, Community, and Culture chez Oxford University Press en I989 ainsi que Contemporary Political Philosophy. An Introduction chez le même éditeur en 1990. 
Guy Laforest est professeur de sciences politiques à l'Université Laval et a dirigé avec Louis Balthazar et Vincent Lemieux la publication du recueil le Québec et la restructuration du Canada I980-I9g2; enjeux et perspectives aux Editions du Septentrion en 1991. Il est aussi l'auteur de Trudeau et la fin d'un rêve canadien chez le même éditeur en 1992.

Josée Legault termine un doctorat en sciences politiques à l'UQAM et est l'auteur de L'invention d'une minorité: les AngloQuébécois aux Editions Boréal en 1992.

Dominique Leydet est professeure au département de philosophie de l'Université d'Ottawa et a publié des articles sur les philosophies morale et politique de Kant, de Hegel et de Habermas.

Kai Nielsen est professeur au département de philosophie de l'Université de Calgary. Il est l'auteur de nombreux ouvrages, dont After the Demise of the Tradition chez Westview Press en 1991 et Equality and Liberty: A Defense of Radical Egalitarianism chez Rowman and Allenheld en 1985.

Michel Seymour est professeur au département de philosophie de l'Université de Montréal et prépare un ouvrage intitulé Ecrits anti-individualistes, à paraître chez Vrin / Bellarmin.

Lukas Sosoe est professeur au departement de philosophie de l'Université de Montréal. Il est notamment l'auteur de Naturalismus Kritik publié chez Karl Alber en 1988 et de Philosophie du droit aux Presses Universitaires de France en 1991, écrit en collaboration avec Alain Renaut.

Daniel Weinstock est chercheur invité au département de philosophie de l'Université de Montréal et a co-dirigé la 
publication d'un recueil de textes sur la pensée politique anglaise des années cinquante intitulé Out of A pathy: the New Left Thirty Years On aux éditions Verso en 1989 . 